\title{
A plug and play approach for the decoration of nanoparticles with recombinant proteins
}

\author{
Enrico Ferrari*,1 \\ ${ }^{1}$ College of Science, University of Lincoln, Brayford Pool, Lincoln LN6 7TS, UK \\ *Author for correspondence: eferrari@lincoln.ac.uk \\ "This article focuses on the idea that designer proteins can be used to form a stable protein \\ corona before injection and exposure to serum proteins."
}

First draft submitted: 27 July 2018; Accepted for publication: 22 August 2018; Published online: 4 October 2018

Keywords: gold nanoparticles $\bullet$ modular assembly $\bullet$ protein corona

As the use of biomacromolecules as therapeutics increases, the challenge of delivering complex biopharmaceuticals, such as those based on proteins and peptides, becomes more pressing [1,2]. Nanocarriers have been traditionally decorated with proteins or peptides for targeting purposes but also for promoting cellular uptake [3] and, in the case of Abraxane, proteins themselves constitute the nanocarrier [4]. Besides the deliberate adsorption or conjugation of proteins onto nanoparticles, nanomedicines administered by intravenous injection will be exposed to high concentrations of proteins in the serum, which readily adsorb on the surface of the nanoparticles forming the so-called protein corona. It is fair to say that unless a nanoparticle has been deliberately engineered to repel proteins, all of them will adsorb proteins at some point during their journey toward the target tissue. It is now well accepted that the formation of a protein layer surrounding the nanoparticle can have significant effect on the biodistribution and the efficacy of the targeting strategy [5-8]. For this reason, the ability to carefully engineer the interface between the nanoparticle and body fluids could play a significant role in future nanomedicines $[9,10]$.

This article focuses on the idea that designer proteins can be used to form a stable protein corona before injection and exposure to serum proteins. The use of proteins that prevent the formation of a serum protein-rich corona and carry targeting or cell penetrating domains could potentially elude opsonization, increase tissue specificity and improve the pharmacokinetics of nanocarriers [5]. However, the controlled adsorption or chemical conjugation of proteins onto nanoparticles is not straightforward and far from universal, due to the remarkable diversity of protein structures and chemistries involved. As a consequence, the conjugation method for each nanoparticle/protein pair would require extensive optimization and this is reflected by the large number of specific functionalization strategies developed so far [11,12]. As the excessive diversification of methods and chemistries in nanocarriers' synthesis could potentially lead to regulatory complications, it is important to study modular strategies to simplify and reduce the number of molecular building blocks required. Such approach was recently reported in the context of modular assembly of proteins on gold nanoparticles [13] and it is detailed below.

\section{Modular assembly of proteins on gold nanoparticles}

The 'plug and play' paradigm in synthetic biology [14] promotes a simple bottom-up approach for the design of artificial biological entities. In general terms, the end user of a plug and play synthetic biology technology should be able, in principle, to combine existing building blocks together to obtain a product, without any need of tedious optimization steps. The modularity of the approach means that some building blocks could be used in more than one process without modification. In the context of protein conjugation to nanoparticles, it can be useful to use an analogy with USB power adaptors to highlight the modular nature of the method described here.

To plug and charge different devices (i.e., the proteins) into country-specific sockets (i.e., the nanoparticles), it would be unpractical to have specifically designed power cords for every device and socket pair. Instead, USB power adaptors offer the possibility to charge any device presenting a USB plug. Similarly, it would be ideal to encode a universal tag (i.e., the USB plug) into a recombinant protein. The tag would allow the end user to 'plug' 
the protein onto any nanoparticle for which an 'adaptor' exists. A molecular equivalent of this adaptor has been recently described [13] for the use with gold nanoparticles. Gold nanoparticles were chosen as a proof-of-concept and for their general relevance in nanomedicine [15]. Recent examples that make use of biomolecules conjugated to gold nanoparticles include delivery of Cas9 ribonucleoprotein and donor DNA for in vivo genome editing [16] and drug delivery across the blood-brain barrier [17].

The part of the 'adaptor' that binds to the gold nanoparticle was the enzyme GST from Schistosoma japonicum. This is a common affinity tag used in protein biochemistry for its ability to bind glutathione-modified resins. It was discovered that GST also adsorbs onto citrate-capped gold nanoparticles with remarkable affinity and the mechanism of binding was described [13]. This involves the formation of covalent bonds between gold and sulfur atoms at cysteine residues (Au-S bonds), which make the binding of GST to gold permanent.

The analog of the adaptor's USB socket was provided by SpyCatcher, which has the specific ability to bind recombinant proteins encoding a SpyTag [18]. SpyCatcher/SpyTag consists of a split protein system engineered from the fibronectin-binding protein FbaB from Streptococcus pyogenes. The two components of the system assemble together spontaneously and form an unusual isopeptidic bond between the two polypeptides. Specifically, the bond forms between the amino group of the side chain of a lysine residue on SpyCatcher and the carboxylic group of the aspartic acid side chain on SpyTag. SpyCatcher was cloned as a C-terminal fusion protein to GST and expressed in Escherichia coli. GST-SpyCatcher, the 'adaptor,' was mixed with $40 \mathrm{~nm}$ gold nanoparticles and adsorbed permanently and stably. A SpyTag recombinant fusion to another molecule of GST was then used to form a second layer of proteins on the nanoparticles. This was obtained again by simple mixing, proving the idea of straightforward, modular and hierarchical decoration of nanoparticles with protein building blocks.

It is important to note that protein adsorption on metal nanoparticles can lead to protein denaturation and potentially compromise protein function [19]. Partial denaturation of GST upon adsorption on both gold and silver was in fact observed [13]. However, as the point of GST in this context was merely to bind firmly to nanoparticles, the actual function to preserve was not the transferase activity of GST but the ability of GST-fused SpyCatcher to bind SpyTag-modified proteins. This example highlights that it could be beneficial to have an intermediate 'sacrificial' layer of proteins directly adsorbed on the solid surface, whereas the second protein layer would be away from the metal, with better preservation of structure and function.

\section{Conclusion}

Overall, the described method showed the excellent gold-binding properties of GST. For example, human serum could not displace GST from gold nanoparticles, suggesting that GST-based anchoring could work in vivo. SpyCatcher/SpyTag system was used as an intermediate for the assembly of a second layer of proteins on top of GST. Both GST binding to gold and SpyTag binding to SpyCatcher were very efficient and were performed by simple mixing in equimolar concentrations and mild conditions. Importantly, both interactions led to the formation of covalent bonds, Au-S bonds on the first protein layer and isopeptidic bonds on the second, thus providing stable conjugates.

This method is particularly suitable for the decoration of gold nanoparticles with recombinant proteins. The 13 amino acids SpyTag can be easily encoded at the N-terminal or C-terminal of a protein of interest. The short length of SpyTag suggests that the method can be very versatile and, for example, could include the possibility to plug into SpyCatcher activated particles any molecule that can be chemically synthesized in association with the 13 amino acids peptide. Using once more the analogy with electronic devices and adaptors, it will be possible to manufacture many different varieties of 'devices' with a SpyTag 'USB plug. For example, recent work on virus-like particles for vaccines used SpyCatcher/SpyTag to display viral epitopes and the modular approach described here may prove useful in this field [20].

Although the activation of nanoparticles using GST-SpyCatcher was mainly described for citrate-capped gold nanoparticles, the method was also applied to silver nanoparticles synthesized in the same way, suggesting that the same building block can be used at least on two materials. The obvious follow-up of this work would be the design and synthesis of new 'adaptors' for nanoparticles other than gold or silver, for example, by fusion of SpyCatcher to peptides that bind specific materials [12]. 
Financial \& competing interests disclosure

The author acknowledges support from the Tom West Analytical Fellowship. The work was in part supported by the European Union's Horizon 2020 research and innovation program (Grant Agreement Number 645684 'Immuno-NanoDecoder'). The author has no other relevant affiliations or financial involvement with any organization or entity with a financial interest in or financial conflict with the subject matter or materials discussed in the manuscript apart from those disclosed.

No writing assistance was utilized in the production of this manuscript.

\section{References}

1. Mitragotri S, Burke PA, Langer R. Overcoming the challenges in administering biopharmaceuticals: formulation and delivery strategies. Nat. Rev. Drug Discov. 13(9), 655-672 (2014).

2. Witting M, Obst K, Friess W, Hedtrich S. Recent advances in topical delivery of proteins and peptides mediated by soft matter nanocarriers. Biotechnol. Adv. 33(6), 1355-1369 (2015).

3. Zhang D, Wang J, Xu D. Cell-penetrating peptides as noninvasive transmembrane vectors for the development of novel multifunctional drug-delivery systems. J. Control. Release 229, 130-139 (2016).

4. Stinchcombe TE. Nanoparticle albumin-bound paclitaxel: a novel Cremphor-EL-free formulation of paclitaxel. Nanomedicine 2(4), 415-423 (2007).

5. Blanco E, Shen H, Ferrari M. Principles of nanoparticle design for overcoming biological barriers to drug delivery. Nat. Biotechnol. 33(9), 941-951 (2015).

6. Bertoli F, Garry D, Monopoli MP, Salvati A, Dawson KA. The intracellular destiny of the protein corona: a study on its cellular internalization and evolution. ACS Nano 10(11), 10471-10479 (2016).

7. Docter D, Westmeier D, Markiewicz M, Stolte S, Knauer SK, Stauber RH. The nanoparticle biomolecule corona: lessons learned challenge accepted? Chem. Soc. Rev. 44(17), 6094-6121 (2015).

8. Walkey $\mathrm{CD}$, Chan WCW. Understanding and controlling the interaction of nanomaterials with proteins in a physiological environment. Chem. Soc. Rev. 41(7), 2780-2799 (2012).

9. Monopoli MP, Åberg C, Salvati A, Dawson KA. Biomolecular coronas provide the biological identity of nanosized materials. Nat. Nanotechnol. 7(12), 779-786 (2012).

10. Canton I, Battaglia G. Endocytosis at the nanoscale. Chem. Soc. Rev. 41(7), 2718-2739 (2012).

11. Avvakumova S, Colombo M, Tortora P, Prosperi D. Biotechnological approaches toward nanoparticle biofunctionalization. Trends Biotechnol. 32(1), 11-20 (2014).

12. Sapsford KE, Algar WR, Berti L et al. Functionalizing nanoparticles with biological molecules: developing chemistries that facilitate nanotechnology. Chem. Rev. 113(3), 1904-2074 (2013).

13. Ma W, Saccardo A, Roccatano D et al. Modular assembly of proteins on nanoparticles. Nat. Commun. 9, 1489 (2018).

14. Medema MH, Breitling R, Bovenberg R, Takano E. Exploiting plug-and-play synthetic biology for drug discovery and production in microorganisms. Nat. Rev. Microbiol. 9(2), 131-137 (2011).

15. Dreaden EC, Alkilany AM, Huang X, Murphy CJ, El-Sayed MA. The golden age: gold nanoparticles for biomedicine. Chem. Soc. Rev. 41(7), 2740-2779 (2012)

16. Lee K, Conboy M, Park HM et al. Nanoparticle delivery of Cas9 ribonucleoprotein and donor DNA in vivo induces homology-directed DNA repair. Nat. Biomed. Eng. 1(11), 889-901 (2017).

17. Gromnicova R, Yilmaz CU, Orhan N et al. Localization and mobility of glucose-coated gold nanoparticles within the brain. Nanomedicine 11(6), 617-625 (2016).

18. Zakeri B, Fierer JO, Celik E et al. Peptide tag forming a rapid covalent bond to a protein, through engineering a bacterial adhesin. Proc. Natl Acad. Sci. USA 109(12), 690-697 (2012).

19. Laera S, Ceccone G, Rossi $\mathrm{F}$ et al. Measuring protein structure and stability of protein-nanoparticle systems with synchrotron radiation circular dichroism. Nano Lett. 11(10), 4480-4484 (2011).

20. Brune KD, Leneghan DB, Brian IJ et al. Plug-and-display: decoration of virus-like particles via isopeptide bonds for modular immunization. Sci. Rep. 6, 19234 (2016). 


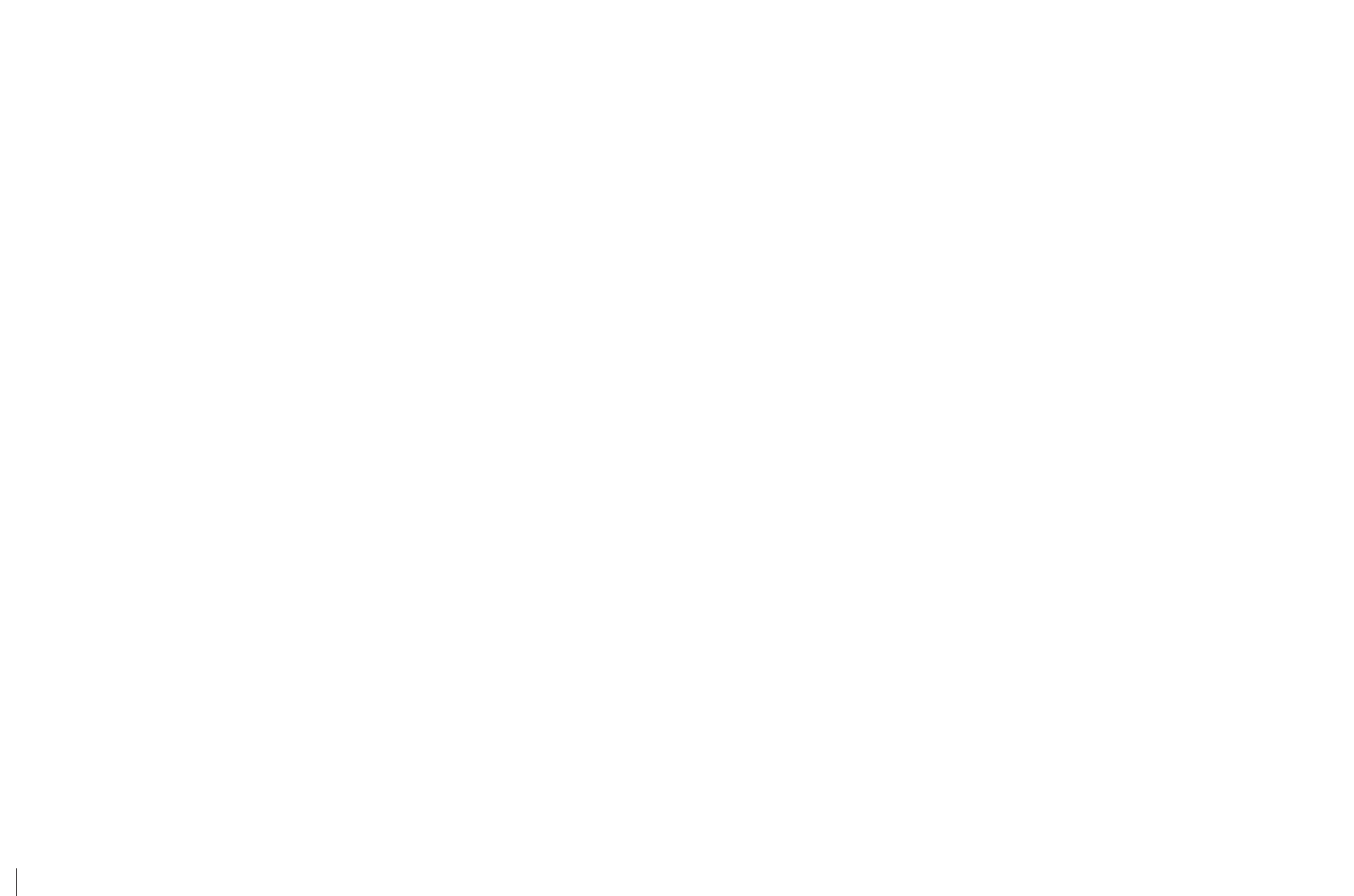

